\title{
Isolation and characterization of endophytic bacteria isolated from the sugarcane cultivated on acrisols of Tay Ninh province, Vietnam
}

\author{
Hoang Minh Tam \\ Dept. Natural Science Teacher Training, Sai Gon University, HCM City, Vietnam \\ Cao Ngoc Diep \\ Dept. Microbiology Biotechnology, Biotechnology R\&D Institute, \\ Can Tho University, Can Tho City, Vietnam
}

\begin{abstract}
Endophytic bacterial diversity in sugarcane plant cultivated on Acrisols of the Tay Ninh province of South Eastern Vietnam was studied. Sugarcane samples were collected from seven sites (districts) of this province. Endophytic bacteria were isolated on LGI medium together with 16S rRNA gene fragments amplified from DNA using eubacterial universal primers (p515FPL and p13B). A total of 139 isolates were isolated from 51 sugarcane samples and all of them have ability of nitrogen fixation and phosphate solubilization as well as IAA biosynthesis but there were 28 isolates having the best characteristics and they were identified as sugarcane endophytes. The sequences from selected endophytic bacteria ( 28 isolates) showed high degrees of similarity to those of the GenBank references strains (between $97 \%$ and $100 \%)$. Among the selected isolates 13 isolates belonged to Bacilli $(\mathbf{4 6 . 4 2 \%})$ whereas 15 isolates belonged to Proteobacteria $(53.57 \%)$ including 6 strains distributed in beta-proteobacteria $(21.42 \%)$, and 9 strains in gamma-proteobacteria (32.14\%). Based on Pi value (nucleotide diversity), Proteobacteria group had the highest Theta values and Theta values (per sequence) from S of SNP for DNA polymorphism were calculated for each group and Proteobacteria group had the highest values in comparison with two groups. From these results showed that many strains as Enterobacter ludwigii TR2, Bacillus amyloliquefasciens CR4e, Bacillus subtilis MT2b, Enterobacter oryzae CT4b, Bacillus subtilis ET1b, Burkholderia tropica CR2d, Bacillus amyloliquefasciens ER4a, Bacillus amyloliquefasciens AT2a, Bacillus subtilis ET5b, and Bacillus amyloliquefasciens BT1 proposed as potential microbial inoculants to continue for researching in the pot experiments and field experiments before producing biofertilizers for sustainable sugarcane production in poor Acrisols in Vietnam because of their benefit and biosafety.
\end{abstract}

Keywords - Acrisols, 16S rRNA Gene Sequence, Endophyte, South-Eastern of Vietnam, Sugarcane

\section{INTRODUCTION}

Sugarcane (Saccharum spp.) is a tropical and sub-tropical crop that can produce sugars and a large amount of chemical fertilizers has been applied to sugarcane to promote early growth in many countries, especially in developing countries [1]. In this sustainable sugarcane production, biological nitrogen fixation (BNF) has replaced chemical fertilizers, and there have been long-term search efforts to identify the $\mathrm{N}_{2}$-fixing bacteria involved in sugarcane production [2][3][4]. Cavalcante and Dobereiner [5] first isolated endophytic $\mathrm{N}_{2}$-fixing bacteria from sugar juice and gave this bacterium its species name, Acetobacter diazotrophicus (now Gluconacetobacter diazotrophicus). Subsequently, several endophytic $\mathrm{N}_{2}$-fixing bacteria, such as Herbaspirillum sp. [6], Pantoea sp. [7], Burkholderia tropica [8], B. unamae [9]. and B. silvatlantica [10], were isolated from sugarcane plants. Endophytes represent a subgroup of the rhizobacterial communities that have the ability to enter the roots of their hosts after the rhizoplane is colonised [11]. Endophytic bacteria colonise the intercellular spaces and the inside of xylem vessels and may promote plant growth directly or indirectly [12][13]. 


\section{International Journal of Innovations in Engineering and Technology (IJIET) \\ http://dx.doi.org/10.21172/ijiet.83.029}

The Tay Ninh province, Vietnam locates from $105^{\circ} 48^{\prime} 43^{\prime \prime}$ to $106^{\circ} 22^{\prime} 48 \square \mathrm{E}$ and from $10^{\circ} 57^{\prime} 08 \square$ to $11^{\circ} 06^{\prime} 16 \square$ $\mathrm{N}$, it is located one of the two regions of South Vietnam situated in the Eastern part of South Vietnam. The soil is mainly acrisols with a $\mathrm{pH}$ range of $3.98-4.56$. They are considered poor nutrient, with an average organic matter of $<1 \%$, a total nitrogen range of $0.07-0.11 \%$, and a very low available phosphorus, cation exchange capacity, exchangeable K and contain more sand in their structure [14]. The eastern South Vietnam and the Mekong Delta are two big sugarcane cultivation regions in the South Vietnam; the sugarcane area occupied $12.7 \%$ with 34,395 ha, 66.5 ton/ha and productivity was $2,329,435$ tons [15]. It has been a general practice to apply $250 \mathrm{~kg} \mathrm{~N} \mathrm{ha}^{-1} \mathrm{yr}^{-1}$ or more in most sugarcane cultivating countries [16] as in sugarcane fields in Spain farmers frequently use high amounts of fertilizer (400-500 kg N ha ${ }^{-1} \mathrm{yr}^{-1}$ ). Interactions and association between microorganism and their host plants may be inhibited by high levels of added fertilizer [17] and many of these bacteria are beneficial to their hosts, and are collectively termed plant growth-promoting rhizobacteria (PGPR). Recent interest has focused particularly upon PGPR that are endophytic (i.e. PGPE), are which have been reported to be associated with important crops such as rice, wheat and sugarcane [18].

In the present work we report characterization of endophytic bacteria isolated from sugarcane collected from acrisols (7 districts) in Tayninh province where is one in two important sugarcane cultivating regions of SouthEastern of Vietnam.

\section{MATERIALS AND METHODS}

\section{A. Plant samples}

The sugarcane fields of seven towns (Ben Cau, Chau Thanh, Duong Minh Chau, Go Dau, Tan Bien, Tan Chau and Trang Bang) in Tay Ninh province were collectted. Fields have been in monoculture for more than five years and sugarcane plants were fertilized with different levels (from 200-500 kg N ha-1 yr-1). Sugarcane plants were collected the whole plant after that roots and stems $(50-\mathrm{cm})$ of sugarcane plant [hybrid variety] (near 6 months old plant) were collected.

\section{B. Isolation of putatively endophytic bacteria}

Bacteria were isolated from the tissues of sugarcane. With the aim of isolating culturable diazotrophic bacteria (and phosphate solubilization and Indole 3 -acetic acid (IAA) production) from the internal tissues, sugarcane plants from hybrid varieties which are planting in these regions, were surface-dissinfected by 30 -s immersion in $70 \%$ ethanol, and then surface-sterilized for 5 min with $4 \%$ sodium hypochrite, and finally washed five times with sterile distilled water [19]. The sterilization process was checked by rolling a peace of stem in an agar plate containing TY-rich medium [20]. The surface-sterilized plants were finally macreated and the suspensions obtained were dilluted to $10-^{6}$ in a sterile solution of $\mathrm{NaCl}(0.9 \%)$. One hundred microliters of the diluted suspensions were used to inoculant vials containing $5 \mathrm{ml}$ of LGI [21]. The vials were incubated at $30^{\circ} \mathrm{C}$ for up to 7 days, and those which showed a growth pellicle were replicated into anew fresh vial, and then streaked onto plates containing the same media for isolation. Finally, individual colonies were obtained, and these were streaked onto TY-rich medium for identification of different morphotypes.

\section{Screening for biofertilizer activities}

The ability to fix $\mathrm{N}_{2}$ was tested on Burk' $\mathrm{N}$-free liquid medium incubating at $30^{\circ} \mathrm{C}$ and the ammonium concentration in medium was measured by Phenol Nitroprusside method after 2,4,6 and 8 days inoculated (DAI) and inorganic phosphate solubilizing ability was tested on NBRIP liquid medium and they were incubated at $30^{\circ} \mathrm{C}$ and the $\mathrm{P}_{2} \mathrm{O}_{5}$ concentration was measured by ammonium molypdate method. The qualitative detection of indole-3-acetic acid (IAA) production was carried out basing on the colorimetric method [22]. Precultures were grown in Burk's N free $(100 \mathrm{ml})$ without tryptophan in $250 \mathrm{~mL}$-flask at $30^{\circ} \mathrm{C}$ on a roller at $100 \mathrm{rpm}$ and samples were taken from at 2, 4, 6, and $8 \mathrm{DAI}$, cell free supernatants were mixed 2:1 with Salkowki reagent $\left(0.01 \mathrm{M} \mathrm{FeCl}_{3}\right.$ in $35 \%$ perchloric acid) and incubated in the dark for $20 \mathrm{~min}$ at RT. IAA-containing solutions were indicated by reddish color with an absorption 


\section{International Journal of Innovations in Engineering and Technology (IJIET) \\ http://dx.doi.org/10.21172/ijiet.83.029}

peak at $530 \mathrm{~nm}$ on Genesys 10uv Thermo Scientific spectrophotometer. Furthermore, siderophore production was assayed by the rhizopheric bacterial isolates according to Schwyn and Neilands [23] using NBRIP medium without tryptophan which was diluted fivefold. The isolates were spot inoculated onto Chrome azurol S agar plates divided into equal sectors, and the plates were incubated at $28^{\circ} \mathrm{C}$ for $48 \mathrm{~h}$. Development of a yellow, orange or violet halo around the bacterial colony was considered to be positive for siderophore production.

\section{D. $16 \mathrm{~S}$ rRNA gene amplification and sequencing}

Bacterial DNA was isolated following published protocols [24]; Amplification of 16S rDNA by PCR was carried out using the primers p515FPL and p13B [25]. The $50 \mu \mathrm{L}$ reaction mixture consisted of $2.5 \mathrm{U}$ Taq Polymerase (Fermentas), $50 \mu \mathrm{M}$ of each desoxynecleotide triphosphate, $500 \mathrm{nM}$ of each primer (Fermentas) and $20 \mathrm{ng}$ DNA. The thermocycling profide was carried out with an initial denaturation at $95^{\circ} \mathrm{C}(5 \mathrm{~min})$ followed by 30 cycles of denaturation at $95^{\circ} \mathrm{C}(30 \mathrm{~s})$, annealing at $55^{\circ} \mathrm{C}(30 \mathrm{~s})$, extension at $72^{\circ} \mathrm{C}(90 \mathrm{~s})$ and a final extension at $72^{\circ} \mathrm{C}(10 \mathrm{~min})$ in C1000 Thermal Cycler (Bio-Rad). Aliquots $(10 \mu \mathrm{l})$ of PCR products were electrophoresed and visualized in 1\% agarose gels using standard electrophoresis procedures. Aliquots $(10 \mu \mathrm{l})$ of PCR products were electrophoresed and visualized in $1 \%$ agarose gels using standard electrophoresis procedures. Partial 16S rRNA gene of selectived isolates in each group were sequenced by MACROGEN, Republic of Korea (dna.macrogen.com). Finally, 16S rRNA sequence of the isolate was compared with that of other microorganisms by way BLAST (http://www.ncbi.nlm.nih.gov/BLAST/Blast.cgi); In the best isolate(s) (high nitrogen fixation and phosphate solubilization ability) and 28 isolates of 7 sites were chosen to sequence and the results were compared to sequences of GenBank based on partial 16S rRNA sequences to show relationships between root-associated bacterial strains [26] and phylogenetic tree were constructed by the neighbor-joining method using the MEGA software version 6.06 based on 1000 bootstraps.

\section{E. SNPs Discovery}

The sequence date from 28 endophytic bacterial isolates were analysed with SeqScape@Software (Applied Biosystem, Foster City, CA, USA). SeqScape is a sequence comparison tool for variant identification, SNP discovery and validation. It considers alignment depth, the base calls in each of the sequences and the associated base quality values. Putative SNPs were accepted as true sequence variants if the quality value exceeded 20. It means a $1 \%$ chance basecall is incorrect.

\section{F. Nucleotide Diversity $(\theta)$}

Nucleotide diversity $(\Theta)$ was calculated by the method described by Halushka et al. [27]

$$
\ominus=\frac{\mathrm{K}}{\mathrm{aL}} \mathrm{a}=\sum_{i=2}^{n} 1 /(i-1)
$$

where $\mathrm{K}$ is the number of SNPs identified in an alignment length, $\mathrm{n}$ is alleles and $\mathrm{L}$ is the total length of sequence (bp).

\section{G. Data Analyses}

Data from ammonium, orthophosphate and IAA concentrations in media were analysed in completely randomized design with three replicates and Duncan test at $\mathrm{P}=0.01$ was used to differentiate between statistically different means using SPSS version 16. 


\section{RESULTS AND DISCUSSION}

\section{A. Bacteria Isolation, Colony Characteristic and Microscopic Examination}

The endophytic bacteria developed in the pellicles of semi solid (in LGI medium) as the previous results of Weber et al. [28], Thu Ha et al. [29] and our previous result [30]. From 51 sugarcane samples of 7 districts [sites] (Ben Cau, Chau Thanh, Duong Minh Chau, Go Dau, Tan Bien, Tan Chau and Trang Bang), 139 isolates were isolated on LGI medium (Table 1).

Table - 1 Sugarcane sample, endophytic bacteria from root and stem of 51 sugarcane samples

\begin{tabular}{|c|c|c|c|c|c|c|c|}
\hline District (site) & Sample number & Root & Ratio (\%) & Stem & Ratio (\%) & Total & Ratio (\%) \\
\hline Ben Cau & 6 & 21 & 77.77 & 6 & 22.23 & 27 & 19.42 \\
\hline Chau Thanh & 12 & 15 & 60.00 & 10 & 40.00 & 25 & 17.98 \\
\hline Duong Minh Chau & 6 & 7 & 58.33 & 5 & 41.67 & 12 & 8.63 \\
\hline Go Dau & 8 & 7 & 41.17 & 10 & 58.82 & 17 & 12.23 \\
\hline Tan Bien & 5 & 9 & 52.94 & 8 & 47.06 & 17 & 12.23 \\
\hline Tan Chau & 8 & 7 & 50.00 & 7 & 50.00 & 14 & 10.07 \\
\hline Trang Bang & 6 & 18 & 66.67 & 9 & 33.33 & 27 & 19.42 \\
\hline Total & 51 & 84 & & 55 & & 139 & 100 \\
\hline
\end{tabular}

They developed very well on the LGI medium from $36-48 \mathrm{~h}$ at $30^{\circ} \mathrm{C}$, their colonies had round-shape, climy, smooth, colourless or milk-color, yellow and some colonies appeared to have much larger size (Figure 1). The cells were observed by SEM and appeared as rod and most of them have motility (Figure 2).
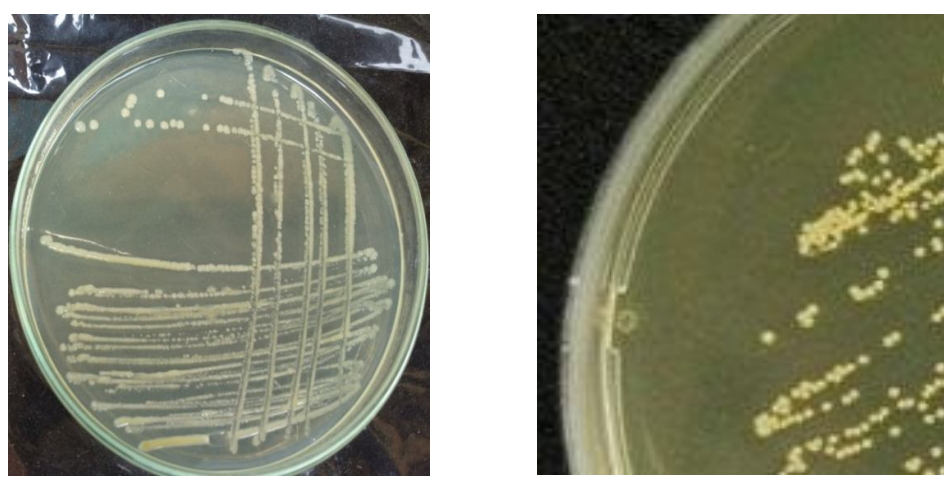

Figure - 1 Colonies of several endophytic isolates from roots and stems of sugarcane

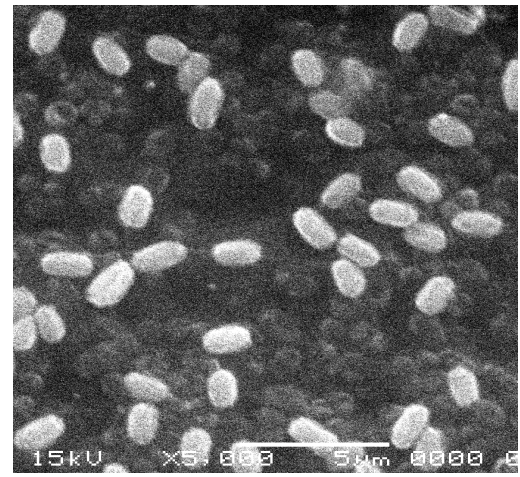

Figure - 2 Electron micrograph of the cells

\subsection{Screening of biofertilizer activities}

All of them (139 isolates) have nitrogen fixation and phosphate solubilization and we only selected 5 isolates having the highest nitrogen fixing ability from each district (Table 2) and the highest phosphate solubilization ability (7 isolates/each district) (Table 3)

Table - 2 Nitrogen fixation of 35 isolates (selected isolates/site)

\begin{tabular}{|l|l|l|l|c|}
\hline \multicolumn{5}{|c|}{ Ben Cau district } \\
\hline Bacterial isolate & \multicolumn{1}{|c|}{ Day 2 Day 4 } & Day 6 & Day 8 \\
\hline BR3d & $0.007 \mathrm{jk}$ & $0.261 \mathrm{~cd}$ & $0.104 \mathrm{~cd}$ & 0.183 cdef \\
\hline
\end{tabular}




\begin{tabular}{|c|c|c|c|c|}
\hline BR1c & $0.006 \mathrm{k}$ & $0.330 \mathrm{a}$ & $0.104 \mathrm{a}$ & 0.148 defgh \\
\hline BR1b & 0.075 ef & $0.125 \mathrm{gh}$ & $0.027 \mathrm{gh}$ & $0.498 \mathrm{a}$ \\
\hline BR3e & $0.120 \mathrm{~cd}$ & $0.334 \mathrm{a}$ & $0.105 \mathrm{a}$ & $0.025 \mathrm{jkl}$ \\
\hline BR1e & $0.059 \mathrm{fg}$ & $0.272 \mathrm{bc}$ & $0.093 \mathrm{bc}$ & $0.072 \mathrm{ijkl}$ \\
\hline control & 0.000 & 0.000 & 0.000 & 0.000 \\
\hline \multicolumn{5}{|c|}{$\mathrm{CV}=15.32 \%$} \\
\hline \multicolumn{5}{|c|}{ Chau Thanh district } \\
\hline CR3a & $0.621 \mathrm{o}$ & $2.419 \mathrm{ab}$ & $0.000 \mathrm{r}$ & $0.311 \mathrm{q}$ \\
\hline CR2e & $1.044 \mathrm{e}$ & $2.087 \mathrm{ab}$ & $0.000 \mathrm{r}$ & $0.341 \mathrm{q}$ \\
\hline CT4b & $0.762 n$ & $2.535 \mathrm{ab}$ & $0.000 \mathrm{r}$ & $0.309 \mathrm{q}$ \\
\hline CR4e & $0.872 \mathrm{i}$ & $2.494 \mathrm{ab}$ & $0.000 \mathrm{r}$ & $0.537 \mathrm{p}$ \\
\hline CT3d & $0.782 \mathrm{~m}$ & $3.578 \mathrm{a}$ & $0.000 \mathrm{r}$ & $0.258 \mathrm{q}$ \\
\hline control & 0.000 & 0.000 & 0.000 & 0.000 \\
\hline \multicolumn{5}{|c|}{$\mathrm{CV}=15.81 \%$} \\
\hline \multicolumn{5}{|c|}{ Duong Minh Chau district } \\
\hline MT2a & $0.037 \mathrm{~g}$ & $0.234 \mathrm{~cd}$ & $0.232 \mathrm{~cd}$ & $0.000 \mathrm{~h}$ \\
\hline MT2b & $0.002 \mathrm{~h}$ & $2.538 \mathrm{a}$ & $0.670 \mathrm{~b}$ & $0.001 \mathrm{~h}$ \\
\hline MR4a & $0.037 \mathrm{~g}$ & $0.146 \mathrm{fg}$ & $0.223 \mathrm{~cd}$ & $0.000 \mathrm{~h}$ \\
\hline MR4c & $0.100 \mathrm{f}$ & $0.221 \mathrm{~cd}$ & $0.224 \mathrm{~d}$ & $0.000 \mathrm{~h}$ \\
\hline MR5a & $0.193 \mathrm{e}$ & $0.303 \mathrm{c}$ & $0.238 \mathrm{~cd}$ & $0.000 \mathrm{~h}$ \\
\hline control & 0.000 & 0.000 & 0.000 & 0.000 \\
\hline \multicolumn{5}{|c|}{$\mathrm{CV}=14.03 \%$} \\
\hline \multicolumn{5}{|c|}{ Go Dau district } \\
\hline GR1c & $0.821 \mathrm{e}$ & $2.469 \mathrm{ab}$ & 0.0001 & $0.258 \mathrm{~h}$ \\
\hline GR1g & $0.829 \mathrm{e}$ & $2.280 \mathrm{ab}$ & $0.000 \quad 1$ & $0.475 \mathrm{f}$ \\
\hline GT3a & $1.247 \mathrm{c}$ & $0.944 \mathrm{~d}$ & 0.0001 & $0.359 \mathrm{~g}$ \\
\hline GR3 & $0.801 \mathrm{e}$ & $2.990 \mathrm{a}$ & 0.0001 & $0.270 \mathrm{~h}$ \\
\hline GT2 & $0.539 \mathrm{f}$ & $0.662 \mathrm{e}$ & $0.022 \mathrm{~h}$ & $0.372 \mathrm{f}$ \\
\hline control & 0.000 & 0.000 & 0.000 & 0.000 \\
\hline \multicolumn{5}{|c|}{$\mathrm{CV}=15.81 \%$} \\
\hline \multicolumn{5}{|c|}{ Tan Bien district } \\
\hline ET1a & $0.392 \mathrm{~d}$ & $0.015 \mathrm{~h}$ & $0.290 \mathrm{~d}$ & $0.352 \mathrm{~d}$ \\
\hline ET2b & $0.149 \mathrm{e}$ & $0.080 \mathrm{f}$ & $0.185 \mathrm{e}$ & $0.722 \mathrm{c}$ \\
\hline ER4a & $0.060 \mathrm{f}$ & $0.039 \mathrm{gh}$ & $0.169 \mathrm{e}$ & $2.032 \mathrm{a}$ \\
\hline ET5b & $1.442 \mathrm{~b}$ & $0.047 \mathrm{~g}$ & $0.166 \mathrm{e}$ & $0.159 \mathrm{e}$ \\
\hline ER5b & $0.049 \mathrm{~g}$ & $0.094 \mathrm{f}$ & $0.289 \mathrm{~d}$ & $0.155 \mathrm{e}$ \\
\hline control & 0.000 & 0.000 & 0.000 & 0.000 \\
\hline \multicolumn{5}{|c|}{$\mathrm{CV}=10.30 \%$} \\
\hline \multicolumn{5}{|c|}{ Tan Chau district } \\
\hline AT1a & $0.025 \mathrm{def}$ & $0.005 \mathrm{~d}$ & $1.365 \mathrm{a}$ & $0.116 \mathrm{e}$ \\
\hline AT1b & $0.003 \mathrm{f}$ & $0.003 \mathrm{~d}$ & 0.182 def & $0.255 \mathrm{a}$ \\
\hline AT2b & $0.031 \mathrm{cde}$ & $0.039 \mathrm{c}$ & $0.779 \mathrm{~b}$ & $0.142 \mathrm{~d}$ \\
\hline AR2 & $0.002 \mathrm{f}$ & $0.189 \mathrm{a}$ & 0.182 def & $0.175 \mathrm{c}$ \\
\hline AR3a & $0.002 \mathrm{f}$ & $0.054 \mathrm{c}$ & $0.065 \mathrm{~h}$ & $0.051 \mathrm{~g}$ \\
\hline control & 0.000 & 0.000 & 0.000 & 0.000 \\
\hline \multicolumn{5}{|c|}{$\mathrm{CV}=9.03 \%$} \\
\hline \multicolumn{5}{|c|}{ Trang Bang district } \\
\hline TR3a & $0.749 \mathrm{e}$ & $0.873 \mathrm{~d}$ & $0.000 \mathrm{i}$ & $0.388 \mathrm{k}$ \\
\hline TR2a & $0.508 \mathrm{gh}$ & $1.216 \mathrm{ab}$ & $0.000 \mathrm{i}$ & $0.313 \mathrm{k}$ \\
\hline TR3c & $0.923 \mathrm{c}$ & $1.356 \mathrm{a}$ & $0.000 \mathrm{i}$ & $0.433 \mathrm{~h}$ \\
\hline TR2e & $0.522 \mathrm{gh}$ & $0.253 \mathrm{kl}$ & $0.459 \mathrm{~h}$ & $0.640 \mathrm{f}$ \\
\hline TT1a & $0.923 \mathrm{c}$ & $0.766 \mathrm{e}$ & $0.572 \mathrm{gh}$ & 0.2101 \\
\hline control & 0.000 & 0.000 & 0.000 & 0.000 \\
\hline \multicolumn{5}{|c|}{$\mathrm{CV}=15.81 \%$} \\
\hline
\end{tabular}

Means within a column followed by the same letter/s are not significantly different at $\mathrm{p}<0.01$

There were a lot of the isolates having highest phosphate solubilization such as CT3a, CR4c (Chau Thanh district), GR1b, GR1d, TR2f, TT1b, TT2a, TT2i (Trang bang district). 
International Journal of Innovations in Engineering and Technology (IJIET)

http://dx.doi.org/10.21172/ijiet.83.029

Table - 3 Phosphate solubilization of 42 isolates (selected isolates/site)

\begin{tabular}{|c|c|c|c|c|}
\hline \multicolumn{5}{|c|}{ Ben Cau district } \\
\hline Bacterial isolate & Day 5 & Day 10 & Day 15 & Day 20 \\
\hline BR1e & $22.80 \mathrm{fgh}$ & $118.25 \mathrm{ab}$ & $146.02 \mathrm{c}$ & $98.79 \mathrm{bc}$ \\
\hline $\mathrm{BR} 2 \mathrm{c}$ & $178.30 \mathrm{a}$ & $115.93 \mathrm{ab}$ & $143.64 \mathrm{c}$ & $59.49 \mathrm{~d}$ \\
\hline BR3c & $40.15 \mathrm{bc}$ & $122.05 \mathrm{a}$ & $191.67 \mathrm{a}$ & $125.34 \mathrm{a}$ \\
\hline BT1 & $21.06 \mathrm{fgh}$ & $102.18 \mathrm{bcd}$ & $114.32 \mathrm{~d}$ & $96.31 \mathrm{c}$ \\
\hline BT3b & $35.66 \mathrm{~cd}$ & $112.87 \mathrm{ab}$ & $161.79 \mathrm{~b}$ & $105.79 \mathrm{~b}$ \\
\hline BT4 & 26.67 ef & $90.97 \mathrm{cde}$ & $123.64 \mathrm{~d}$ & $59.67 \mathrm{~d}$ \\
\hline $\mathrm{DC}$ & 0.00 & 0.00 & 0.00 & 0.00 \\
\hline \multicolumn{5}{|c|}{$\mathrm{CV}=6.61 \%$} \\
\hline \multicolumn{5}{|c|}{ Chau Thanh district } \\
\hline CT1 & $166.49 \mathrm{q}$ & 205.18 a & $282.91 \mathrm{p}$ & $285.02 \mathrm{k}$ \\
\hline CR4b & $150.53 \mathrm{q}$ & $155.92 \mathrm{q}$ & $378.46 \mathrm{a}$ & 278.661 \\
\hline CT3a & $196.87 \mathrm{~m}$ & $222.43 \mathrm{e}$ & 292.32 o & $301.51 \mathrm{e}$ \\
\hline CR4c & $336.46 \mathrm{ab}$ & $256.85 \mathrm{a}$ & $323.84 \mathrm{k}$ & $329.38 \mathrm{ab}$ \\
\hline CT3d & $83.72 \mathrm{~s}$ & 174.55 o & $240.90 \mathrm{q}$ & $217.88 \mathrm{~m}$ \\
\hline CR3a & $135.43 \mathrm{q}$ & $200.85 \mathrm{j}$ & $116.86 \mathrm{r}$ & $285.37 \mathrm{j}$ \\
\hline Control & 0.00 & 0.00 & 0.000 & 0.00 \\
\hline \multicolumn{5}{|c|}{$\mathrm{CV}=12.04 \%$} \\
\hline \multicolumn{5}{|c|}{ Duong Minh Chau district } \\
\hline MR2 & $7.86 \mathrm{ab}$ & 5.89 efg & $43.14 \mathrm{gh}$ & $55.41 \mathrm{a}$ \\
\hline MR4a & $6.29 \mathrm{~cd}$ & $16.61 \mathrm{a}$ & $248.32 \mathrm{a}$ & $12.16 \mathrm{ef}$ \\
\hline MR4c & $8.86 \mathrm{a}$ & $12.18 \mathrm{~b}$ & $155.25 \mathrm{~b}$ & $29.88 \mathrm{~b}$ \\
\hline MT1b & $6.16 \mathrm{de}$ & $9.73 \mathrm{bcd}$ & $80.38 \mathrm{de}$ & $17.16 \mathrm{~cd}$ \\
\hline MT2a & $7.57 \mathrm{abc}$ & $11.64 \mathrm{~b}$ & $150.75 \mathrm{~b}$ & $25.76 \mathrm{~b}$ \\
\hline MT2b & $6.81 \mathrm{bcd}$ & $6.84 \mathrm{def}$ & $43.74 \mathrm{gh}$ & $8.32 \mathrm{fg}$ \\
\hline Control & 0.00 & 0.00 & 0.00 & 0.00 \\
\hline \multicolumn{5}{|c|}{$\mathrm{CV}=12.60 \%$} \\
\hline \multicolumn{5}{|c|}{ Go Dau district } \\
\hline GT3a & $116.14 \mathrm{q}$ & $115.01 \mathrm{q}$ & $326.27 \mathrm{j}$ & $270.50 \mathrm{n}$ \\
\hline GT1b & $149.92 \mathrm{q}$ & $167.62 \mathrm{q}$ & $333.33 \mathrm{~h}$ & $204.46 \mathrm{q}$ \\
\hline GR1b & $118.23 \mathrm{q}$ & 190.731 & $354.12 \mathrm{f}$ & $222.30 \mathrm{q}$ \\
\hline GR1d & $343.29 \mathrm{a}$ & $178.04 \mathrm{n}$ & $228.79 \mathrm{q}$ & $165.01 \mathrm{q}$ \\
\hline GT1c & $68.84 \mathrm{q}$ & $254.43 \mathrm{abc}$ & $365.90 \mathrm{~d}$ & $306.55 \mathrm{abc}$ \\
\hline GR1e & $176.30 \mathrm{o}$ & $256.38 \mathrm{ab}$ & $336.22 \mathrm{~g}$ & 387.72 a \\
\hline $\mathrm{DC}$ & 0.00 & 0.00 & 0.00 & 0.00 \\
\hline \multicolumn{5}{|c|}{$\mathrm{CV}=12.04 \%$} \\
\hline \multicolumn{5}{|c|}{ Tan Bien district } \\
\hline ET1b & $50.27 \mathrm{gh}$ & $88.31 \mathrm{abc}$ & $313.14 \mathrm{~b}$ & $252.33 \mathrm{~b}$ \\
\hline ET2a & $125.86 \mathrm{a}$ & $93.72 \mathrm{a}$ & $360.46 \mathrm{a}$ & $349.53 \mathrm{a}$ \\
\hline ET3 & $125.28 \mathrm{a}$ & $92.90 \mathrm{ab}$ & $228.71 \mathrm{c}$ & $67.13 \mathrm{c}$ \\
\hline ER4b & $64.09 \mathrm{def}$ & $93.72 \mathrm{a}$ & $320.44 \mathrm{~b}$ & $255.06 \mathrm{~b}$ \\
\hline ER2a & $38.91 \mathrm{hi}$ & $70.24 \mathrm{c}$ & $181.76 \mathrm{f}$ & $122.14 \mathrm{f}$ \\
\hline ET5c & $126.14 \mathrm{a}$ & $93.72 \mathrm{a}$ & $208.52 \mathrm{de}$ & $147.79 \mathrm{~cd}$ \\
\hline Control & 0.00 & 0.00 & 0.00 & 0.00 \\
\hline \multicolumn{5}{|c|}{$\mathrm{CV}=4.86 \%$} \\
\hline \multicolumn{5}{|c|}{ Tan Chau district } \\
\hline AT1b & $29.31 \mathrm{f}$ & $125.72 \mathrm{a}$ & 123.72 ef & $244.06 \mathrm{~b}$ \\
\hline AT2b & $77.19 \mathrm{~d}$ & $76.04 \mathrm{f}$ & $152.91 \mathrm{a}$ & $213.46 \mathrm{c}$ \\
\hline AT2c & $222.80 \mathrm{a}$ & $124.44 \mathrm{a}$ & $145.80 \mathrm{ab}$ & $178.88 \mathrm{~d}$ \\
\hline AR2 & $107.16 \mathrm{c}$ & $105.60 \mathrm{c}$ & $145.33 \mathrm{ab}$ & $402.30 \mathrm{a}$ \\
\hline AR3c & $137.88 \mathrm{~b}$ & $123.99 \mathrm{a}$ & $134.44 \mathrm{~cd}$ & $95.61 \mathrm{~h}$ \\
\hline AR4b & $53.98 \mathrm{e}$ & $125.72 \mathrm{a}$ & $145.80 \mathrm{ab}$ & $219.78 \mathrm{bc}$ \\
\hline control & 0.00 & 0.00 & 0.00 & 0.00 \\
\hline \multicolumn{5}{|c|}{$\mathrm{CV}=5.71 \%$} \\
\hline
\end{tabular}




\begin{tabular}{|c|c|c|c|c|}
\hline AT1b & $1.878 \mathrm{~b}$ & $3.994 \mathrm{c}$ & $3.065 \mathrm{~b}$ & $3.541 \mathrm{~d}$ \\
\hline AT2a & $2.041 \mathrm{~b}$ & $2.543 \mathrm{e}$ & $3.944 \mathrm{a}$ & $6.125 \mathrm{a}$ \\
\hline AT2b & $1.895 \mathrm{~b}$ & $5.810 \mathrm{a}$ & $1.537 \mathrm{f}$ & $6.351 \mathrm{a}$ \\
\hline AR3b & $1.985 \mathrm{~b}$ & $2.978 \mathrm{~d}$ & $4.084 \mathrm{a}$ & $5.733 \mathrm{~b}$ \\
\hline AR4b & $1.247 \mathrm{~cd}$ & $5.862 \mathrm{a}$ & $4.066 \mathrm{a}$ & $1.553 \mathrm{~h}$ \\
\hline Control & 0.000 & 0.000 & 0.000 & 0.000 \\
\hline \multicolumn{5}{|c|}{$\mathrm{CV}=4.49 \%$} \\
\hline \multicolumn{5}{|c|}{ Trang Bang district } \\
\hline TR2h & $8.231 \mathrm{q}$ & 8.333 о & $8.155 \mathrm{i}$ & $8.441 \mathrm{e}$ \\
\hline TR3d & $8.282 \mathrm{q}$ & $9.697 \mathrm{~h}$ & $9.226 \mathrm{i}$ & $6.290 \mathrm{q}$ \\
\hline TR2d & $9.718 \mathrm{k}$ & $7.020 \mathrm{q}$ & $10.417 \mathrm{i}$ & $6.667 \mathrm{p}$ \\
\hline TR1d & $10.897 \mathrm{~g}$ & $11.667 \mathrm{a}$ & $9.464 \mathrm{i}$ & $9.247 \mathrm{ab}$ \\
\hline TT2e & $9.916 \mathrm{j}$ & $10.309 \mathrm{~g}$ & $18.761 \mathrm{ab}$ & $7.738 \mathrm{i}$ \\
\hline Control & 0.000 & 0.000 & 0.000 & 0.000 \\
\hline
\end{tabular}

Means within a column followed by the same letter/s are not significantly different at $\mathrm{p}<0.01$

Ratio (\%) bacterial isolate producing siderophores was $23.02 \%$, number of endophytic bacterial isolate producing siderophores (8/27) isolated on plant sugarcane cultivating on Ben Cau was the highest (Table 5) )Figure 3) but endophytes at Duong Minh Chau site have no siderophores

Table - 5 Quantative and ratio (\%) bacterial isolates produced siderophores

\begin{tabular}{|c|c|c|c|}
\hline District (site) & Isolate has Siderophore & Total of isolate & Ratio (\%) \\
\hline Ben Cau & 8 & 27 & 29.62 \\
\hline Chau Thanh & 7 & 25 & 28.00 \\
\hline Duong Minh Chau & 0 & 12 & 0 \\
\hline Go Dau & 5 & 17 & 29.41 \\
\hline Tan Bien & 3 & 17 & 17.64 \\
\hline Tan Chau & 5 & 14 & 35.71 \\
\hline Trang Bang & 4 & 27 & 14.81 \\
\hline
\end{tabular}
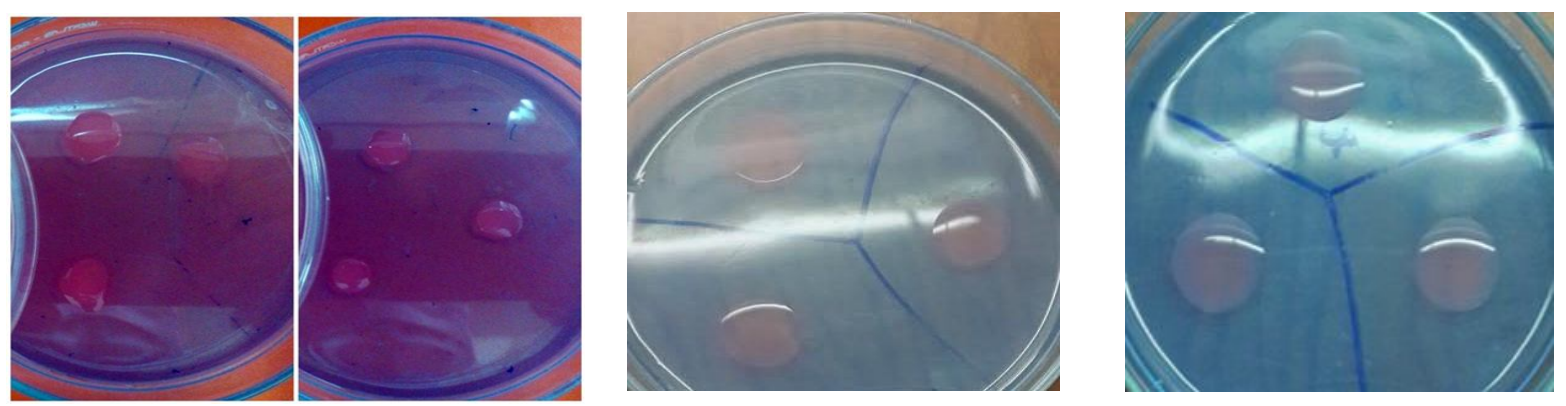

Figure - 3 Bacterial isolates made a yellow, orange halo round well contaning bacterial liquid on CAS agar after $48 \mathrm{~h}$ incubation

Based on the characteristics as high nitrogen fixation, phosphate solublization, IAA and siderophores, 28 good isolates were chosen to identify with universal primers p515FPL and p13B and sequencing as GT1e, TR3c, MR4a, CT4b, TR2e, MR4c, TR2c, GT3b, ER4a, AT2a, BR1e, ET5b, BT1, AT2b, AR4b, ET1b, MT1b, CR2d, CT4a, GT2, TR3a, TR2a, CR4e, TT1a, GR1g, MT2b, GT3a, GR3.

The fragments of 900 bp 16S rRNA were obtained from PCR with p515FPL and p13B primers and sequencing. Homology searches of 16S rRNA gene sequence of selected strain in GenBank by BLAST revealved that they had similarity to sequences of Bacilli (13/28 isolates), 6 isolates belonged to Betaproteobacteria, 9 strains being Gammaproteobacteria (Figure 4) (Table 6). 


\section{International Journal of Innovations in Engineering and Technology (IJIET) http://dx.doi.org/10.21172/ijiet.83.029}

Table - 6 Phylogenetic affiliation of isolates on the basis of 16S rRNA genes sequences by using BLAST programme in the GenBank database based on sequences similarity

\begin{tabular}{|c|c|c|}
\hline Taxonomic group and strain & Closest species relative & Similarity $(\%)$ \\
\hline \multicolumn{3}{|l|}{ Bacilli } \\
\hline \multirow[t]{2}{*}{ CR4e } & Bacillus amyloliquefaciens strain TCCB001 (KC755040) & 99 \\
\hline & Bacillus licheniformis strain N27-EC (KF768851) & 99 \\
\hline \multirow[t]{2}{*}{ GR1g } & Bacillus methylotrophicus strain SDI-57 (KT021536) & 99 \\
\hline & Bacillus subtilis strain SCKB1443 (KM922582) & 99 \\
\hline \multirow[t]{2}{*}{ TT1a } & Bacillus amyloliquefaciens strain TCCB001 (KC755040) & 100 \\
\hline & Bacillus licheniformis strain N27-EC (KF768851) & 100 \\
\hline \multirow[t]{2}{*}{ MT2b } & Bacillus subtilis subsp. inaquosorum strain OUN3RKSP (KM875553) & 99 \\
\hline & Bacillus amyloliquefaciens, strain CEES, isolate CEES\#3 (LN827664) & 99 \\
\hline \multirow[t]{2}{*}{ MT1b } & Bacillus methylotrophicus strain SB-19 (KU522192) & 99 \\
\hline & Bacillus subtilis strain S5-1 (KJ496375) & 99 \\
\hline \multirow[t]{2}{*}{ MR4c } & Bacillus sp. DU27(2010) (HM567081) & 99 \\
\hline & Bacillus thuringiensis strain SY-BT11 (KY127450) & 99 \\
\hline \multirow[t]{2}{*}{ ET5b } & Bacillus subtilis strain SHHR2-10 (KT216596) & 99 \\
\hline & Bacillus siamensis strain RD_AZPIS_04 (KU597583) & 99 \\
\hline \multirow[t]{2}{*}{ ER4a } & Bacillus amyloliquefaciens strain 3EC2C2 (EU304922) & 100 \\
\hline & Bacillus licheniformis strain 3EC4A14 (EU304939) & 100 \\
\hline \multirow[t]{2}{*}{ BT1 } & Bacillus amyloliquefaciens strain 3EC2C2 (EU304922) & 100 \\
\hline & Bacillus licheniformis strain 3EC4A14 (EU304939) & 100 \\
\hline \multirow[t]{2}{*}{ AT2a } & Bacillus amyloliquefaciens strain 3EC2C1 (EU304921) & 100 \\
\hline & Bacillus atrophaeus strain 3EC1B2 (EU304906) & 100 \\
\hline \multirow[t]{2}{*}{ ET1b } & Bacillus subtilis strain 3EC6B4 (EU304967) & 100 \\
\hline & Bacillus licheniformis strain 3EC4A9 (EU304934) & 100 \\
\hline \multirow[t]{2}{*}{ AT2b } & Bacillus megaterium strain S-5 (KT619080) & 99 \\
\hline & Bacillus aryabhattai strain D7 (MF109131) & 99 \\
\hline \multirow[t]{2}{*}{ MR4a } & Bacillus subtilis strain F-36 (KT027687) & 99 \\
\hline & Bacillus sp. DU176(2010) (HM567057) & 99 \\
\hline \multicolumn{3}{|l|}{ Betaproteobacteria } \\
\hline \multirow[t]{2}{*}{ GT1e } & Burkholderia tropica strain 183 (KP969068) & 99 \\
\hline & Burkholderia tropica strain 171 (KP969066) & 99 \\
\hline \multirow[t]{2}{*}{ CR2d } & Burkholderia tropica strain PPe5 (KP974788) & 99 \\
\hline & Paraburkholderia tropica strain S23-9 (KU049652) & 99 \\
\hline \multirow[t]{2}{*}{ GR3 } & Burkholderia vietnamiensis strain BTF5 (KY939774) & 99 \\
\hline & Burkholderia sp. strain BAAr-214 (KY810677) & 99 \\
\hline \multirow[t]{2}{*}{ TR3a } & Burkholderia sp. 2335 (JX174212) & 99 \\
\hline & Burkholderia tropica strain R5-138 (JQ659722) & 99 \\
\hline \multirow[t]{2}{*}{ CT4a } & Burkholderia tropica strain BRUESC674 (KT390891) & 99 \\
\hline & Burkholderia sp. W20 (KC853184) & 99 \\
\hline TR3c & Advenella kashmirensis, strain cv4 (LN870300) & 99 \\
\hline & Advenella kashmirensis strain w13003 (KF147541) & 99 \\
\hline Gammaproteobacteria & & \\
\hline TR2e & Enterobacter cloacae strain Z177 (KF835819) & 99 \\
\hline & Enterobacter asburiae strain PWX1 (KU942481) & 99 \\
\hline GT3a & Enterobacter $\mathrm{sp}$. PtB20 $(\mathrm{KX} 250252)$ & 99 \\
\hline & Enterobacter aerogenes strain KNUC5009 (JQ682634) & 99 \\
\hline TR2c & Enterobacter cloacae strain F16 (KX896752) & 99 \\
\hline & Enterobacter hormaechei subsp. oharae strain DSM 16687 (CP017180) & 99 \\
\hline TR2a & Enterobacter ludwigii strain SWA1 (KF938661) & 99 \\
\hline & Enterobacter cloacae strain NF708 (KJ558518) & 99 \\
\hline GT2 & Enterobacter aerogenes strain LSRC164 (JF772083) & 99 \\
\hline & Kosakonia oryzae strain P-9 (KF479042) & 99 \\
\hline CT4b & Kosakonia oryzae strain VRBG-49 (KR265442) & 99 \\
\hline & Enterobacter oryzae strain $22(\mathrm{KC} 843380)$ & 99 \\
\hline GT3b & Enterobacter oryzae strain $22(\mathrm{KC} 843380)$ & 99 \\
\hline & Kosakonia oryzae isolate B052 (KT275833) & 99 \\
\hline
\end{tabular}




\begin{tabular}{|l|c|c|}
\hline AR4b & Serratia marcescens strain B3R3 (KJ513465) & 97 \\
\hline BR1e & Serratia nematodiphila strain YS8 (KY887776) & 97 \\
\hline & Klebsiella pneumoniae strain BISR-TS19 (KP996204) & 99 \\
\hline
\end{tabular}

A neighbor-joining analysis of phylogenetic tree in these isolates showed in the two clusters: cluster A was big cluster included two clusters: cluster A1 composed of cluster A11 with cluster A111 having two strains Kosakonia oryzae GT2 and Burkholderia tropica TR3a with close relationship (100\%) even though they are Betaproteobacteria and Gammaproteobacteria, respectively and they had relationship with strain Enterobacter ludwigi TR2a while two strains Bacillus amyloliquefasciens CR4e and Bacillus amyloliquefasciens TT1a related closely even though they were isolated at Chau Thanh district (CR4e) and Trang Bang district (TT1a) in cluster A112. Cluster A121 with cluster A1211 comprised of two strains Enterobacter sp. GT3a and Bacillus sp. MR4c and cluster A1221 with two strains Enterobacter cloacae TR2c and Enterobacter oryzae GT3b related closely even though they were isolated two sites far from many kilometers (Trang Bang district $=$ TR2c and Go Dau district $=$ GT3b). Cluster A12 with two strains Bacillus methylotrophicus GR1g and Burkholderia vietnamiensis GR3 had close relationship although they are bacteria Gram-postive (B. methylotrophicus GR1g) and bacteria Gram-negative (Burkholderia vietnamiensis GR3). Cluster A2 had two clusters: cluster A21 with three strains Burkholderia tropica GT1a, Advenella kashminensis TR3c and Bacillus subtillus MR4a had close relationship in small cluster (cluster A211), and two strains Enterobacter oryzae CT4b and Enterobacter cloacae TR2e had a close relatioship (100\%) even though they were isolated at Chau Thanh district (strain CT4b) and at Trang Bang district (strain TR2e) in cluster A212.

Cluster A22 had two clusters: cluster A221 with two strains Serratia marcescens AR4 and Bacillus subtillis ET1b related very closely while three strains Bacillus methylotrophicus MT1b, Burkholderia tropica CR2d and Burkholderia tropica CT4a had a relationship very close (100\%) in cluster A222.

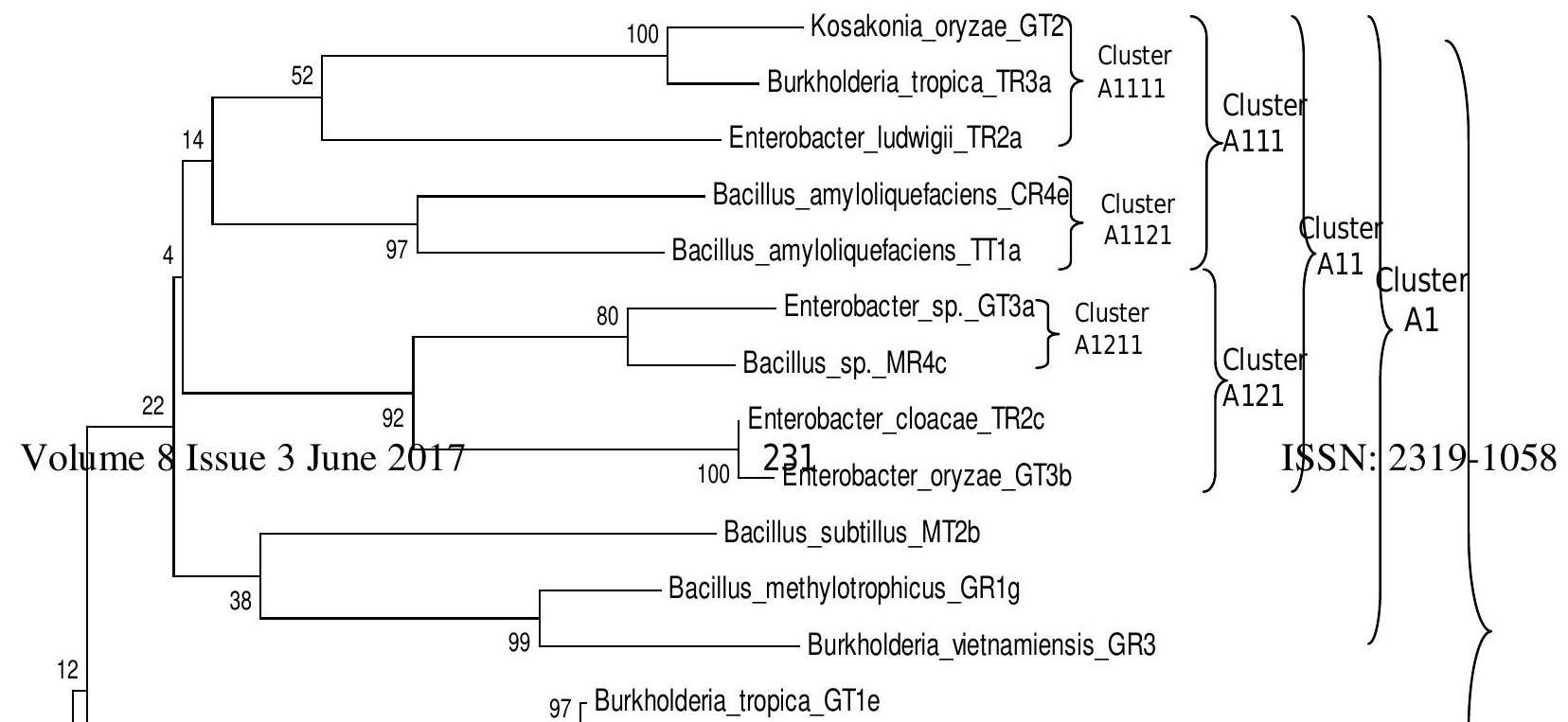



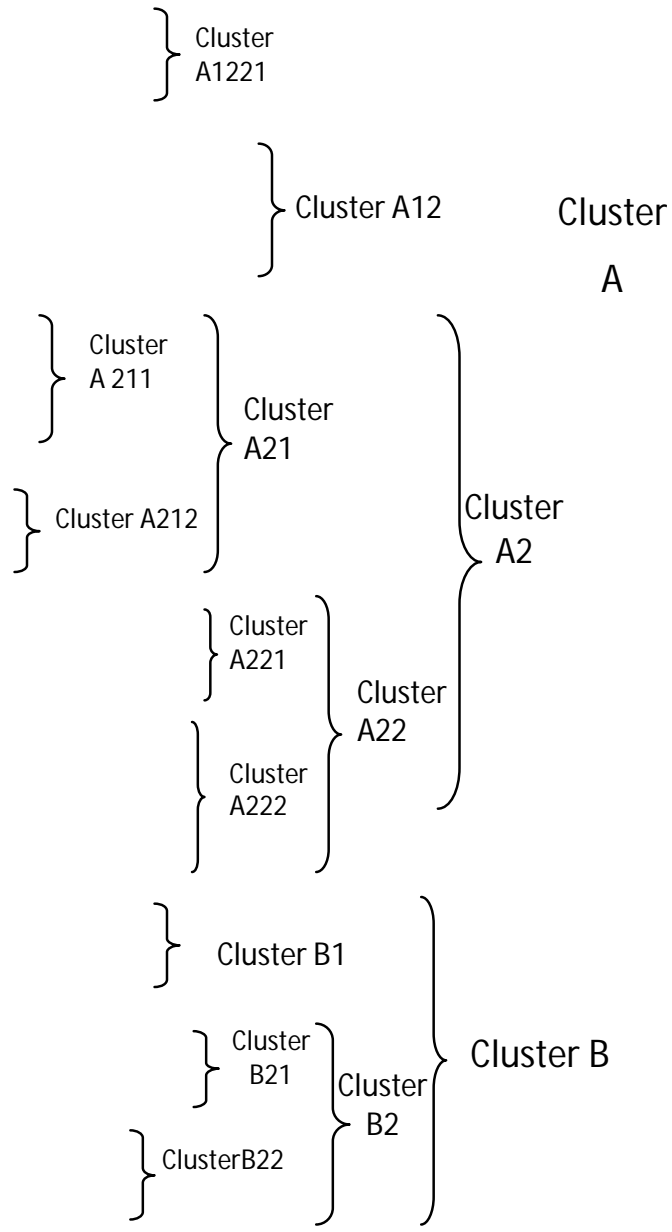

Figure - 4 Phylogenetic tree showing the relative position of endophytic bacterial isolates by the neighbor-joining method of complete 16S rRNA sequence (p515FPL primer).

Bootstrap values of 1000 replicates are shown at the nodes of the trees.

Cluster B was a simple cluster with cluster B1 including two strains Bacillus amyloliquefasciens ER4a and Bacillus amyloliquefasciens AT2a had a close relationship (100\%) and cluster B2 had small clusters: cluster B21 with two strains Klebsiella pneumoniae BR1e and Bacillus subtilis ET5b related closely related while cluster B22 with two strains Bacillus amyloliquefasciens BT1 and Bacillus megaterium AT2b also had a close relationship.

Except two strains Bacillus amyloliquefasciens ER4a and Bacillus amyloliquefasciens AT2a having a relationship very closely (100\%). In other cases, between two endophytic bacterial strains had a relationship in sugarcane plant but they were not sure same species, same genus or same gram-positive/gram-negative.

These results showed that the strains had a relationship very close even though they were isolated from tissue of sugarcane of distance sites/districts.

Among 28 strains, there were 19 strains having length nucleotide (over 600) and Theta values (per sequence) from S of SNP for DNA polymorphism were calculated for Each group, and Gammaproteobacteria group had the 
highest values as comparison with Betaproteobacteria and Bacilli (Table 7). Nucleotide polymorphism can be measured by many parameters, such as halotypes (genes) diversity, nucleotide diversity, (Pi), Theta ( $\square$ )(per group) etc In this study, nucleotide diversity was estimated by Theta ( $\square$ ), the number of segregating sites [31], and its standard deviation $(\mathrm{S} \square$ ). These parameters were estimated by DNA Sequence Polymorphism software version 4.0 [32]. Pi values explained nucleotide diversity of sequences for each gene, the highest values, more diversity among groups.

Table - 7 Genetic diversity of 21 strains

\begin{tabular}{lccc}
\hline & Nucleotie diversity & Theta (per site) from Eta & Theta (per site) from S ( $\square$ ) \\
\hline 21 strains & 0.71984 & $0.83199 \pm 0.097$ & $0.28611 \pm 0.0095$ \\
\hline
\end{tabular}

Primer p515FPL 5'-GTGCCAGCAGCCGCGTAA-3'

Primer p13B 5'-AGGCCCGGGAACGTATTCAC-3'

The endophytic bacteria have been studied and described as beneficial bacteria with Bacilli, Gammaproteobacteria and Betaproteobacteria presented on LGI medium and it occupied $47 \%, 32 \%$ and $21 \%$ respectively in the total of 28 strains according to our result (Figure 5).

Figure - 5 The proportion of group and they distributed in two clusters

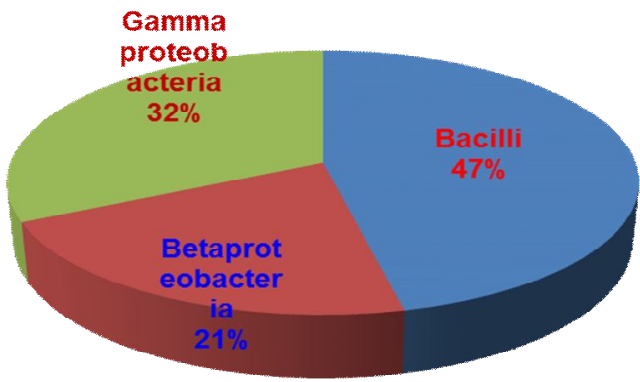

Sugarcane (Sacharum officinarum L.) is grown in more than 120 countries, mainly in Brasil and India [3]. Several genera of diazotrophic, endophytic bacteria were isolated from roots, stems and leaves of sugarcane: Enterobacter, Pantoea, Klebsiella, Pseudomonas, Herbaspirillum, Gluconacetobacter, Azosipirillum [33][34][35][36]. Biological Nitrogen Fixation (BNF), the reduction of $\mathrm{N}_{2}$ to ammonium, causes great transformations in the Nitrogen cycle, BNF is carried out by prokaryotes only, bacteria and archae, which include most of the bacterial phylogenetic groups [37].

In sugarcane, most of the research on endophytic bacteria has focused on diazotrophs, of which the main representatives are Gluconacetobacter diazotrophicus, Herbaspirillum spp. [38][5] and Azospirillum amazonense [39]. However, the presence of diazotroph among the total of bacteria in sugarcane tissues seems to be low in Indian sugarcane [40]. Magnani et al. [41] discovered 32 endophytic bacterial isolates in Brazilian sugarcane (stem and leaf tissues) and 14 strains were classified as the Enterobacteriaceae (Gamma-proteobacteria), among which were Enterobacter (9 strains), Pantoea (3 strains), Kluyvera (1 strain) and Klebsiella (1 strain), based on 16S rRNA sequences. Members of the Enterobactericeae family (Gamma-proteobacteria) are frequntly described as rhizosphere colonizers of sugarcane and other grasses [42]. This class includes Enterobacter cloacae and Pantoea agglomerans (formerly Erwinia herbicola) [43]. Many studies have reported the endophytic presence of Enterobacteriaceae members in various crop species [44]. In our previous results in Dong Nai province showed that 10 endophytic bacterial strains in sugarcane cultivating on latosols and acrisols of two sites (Dinhquan and Trangbom), Dong Nai province, the southeast of Vietnam belonged to Proteobacteria (Gram-negative bacteria) with $50 \%$ strains are Gammaproteobacteria among two strains, Enterobacter oryzae LT7 and Pantoea agglomerans T12, and P. 
agglomerans has been described to be an important corn and wheat endophyte [45], and it has also been isolated from potato stems [46], rice seeds [47] and citrus leaves [48]. Many studies have shown the potential of Pantoea spp. For systemetic resistance induction [49] and protection against pests and plant-pathogenic microorganisms [50]. Additionally, these bacteria may induce plant growth by increasing the nitrogen supply in nonsymbiotic associations [51], solubilizing phosphorus [52] and stimulating phytohormone production [53] and recent result of Quecine et al. [54] applied Pantoea agglomerans 33.1 as sugarcane growth promotion successfully. Besides that, Jha and Kumar [55] also identified a novel plant growth promoting endophytic bacterium Achromobacter xylosoxidans from wheat plant and our results also discovered Achromobacter xylosoxidans T16 having good characteristics as high nitrogen fixation, phosphate solubilization and IAA biosynthesis. In our previous results, 4 good strains as Enterobacter oryzae LT7, Achromobacter xylosoxidans T16, Achromobacter insolitus R15b and Pantoea agglomerans T12 to evaluate their effects on sugarcane cultivated on acrisols in pot-experiment and the field trial [30]. Based on biosafety and good characteristics as nitrogen fixation, phosphate solubilization, IAA synthesis, siderophores production, Enterobacter ludwigii TR2, Bacillus amyloliquefasciens CR4e, Bacillus subtilis MT2b, Enterobacter oryzae CT4b, Bacillus subtilis ET1b, Burkholderia tropica CR2d, Bacillus amyloliquefasciens ER4a, Bacillus amyloliquefasciens AT2a, Bacillus subtilis ET5b, and Bacillus amyloliquefasciens BT1 selected to evaluate their effects on pot-experiment and field trials before producing biofertilizers for sustainable sugarcane caultivation on acrisols of Tay Ninh province.

\section{CONCLUSION}

From 51 field-grown sugarcane samples on acrisols in 7 districts of Tay Ninh province of the South-Eastern Vietnam, 139 isolates were isolated and identified as sugarcane endophytes, 28 isolates having good plant growth promotion from 7 sites were chosen to analyse their relationship. The results showed that bacterial diversity was very high; 10 good strains selected to evaluate their effects on sugarcane in pot-experiments and field experiments.

\section{ACKNOWLEDGEMENTS}

The authors thank the helpfulness of Microbiology BSc. Students and technicians in the Environment Microbiology Laboratory, Biotechnology R\&D Institute, Can Tho University, Vietnam; especially Associate Professor Dr. TRUONG TRONG NGON, Head of Molecular Biotechnology Department, Biotechnology R\&D Institute, Can Tho University, Vietnam for analysing molecular data, Ms. NGUYEN THI XUAN MY in the laboratory expriment and Ms. DAO THI MINH CHAU, Environment Microbiology Laboratory, Biotechnology R\&D Institute, Can Tho University, Vietnam for grammartical english.

REFERENCE

[1] N. Thaweenut, Y. Hachisuka, S. Ando, S. Yanagisawa and T. Yoneyama, "Two season' study on nifH gene expression and nitrogen fixation by diazotrophic endophytes in sugarcane (Saccharum spp. hybrids): expression of nifH genes similar to those of rhizobia," Plant Soil, vol. 338, pp. 435-449, 2011.

[2] J. Dobereiner, "Biological nitrogen fixation in the tropics: social and economic contributions," Soil Biol. Biochem,., vol. 29, pp.771-774, 1997.

[3] J.L. Baldani, V.M. Reis, V.L.D. Baldani and J. Dobereiner, "A brief story of nitrogen fixation in sugarcane - reasons for success in Brasil," Funct. Plant. Biol., vol.29, pp.417-423, 2002.

[4] R.M. Boddey, S. Urguillar, B.J.R. Reis, and V. Reis,"Endophytic nitrogen fixation in sugarcane: present knowledge and future applications," Plant Soil., vol. 252, pp: 139-149, 2003.

[5] V.A. Cavalcante and J. Dobereiner, "A new acid tolerant nitrogen fixing bacterium associated with sugarcane”. Plant and Soil, vol, 108, pp.23-31.

[6] F.L.Olivares, E.K.James, J.I.Baldani and J. Dobereiner, “Infection of molted stripe disease susceptible and resistant varieties of sugarcane by the endophytic diazotroph Herbaspirillum, New Phytologist., vol. 135, pp.723-737, 1997. 


\section{International Journal of Innovations in Engineering and Technology (IJIET) \\ http://dx.doi.org/10.21172/ijiet.83.029}

[7] F.G. Loiret, E. Ortega, D. Kleiner, P. Ortega-Rodes, R. Rodés, Z. Dong, “A putative new endophytic nitrogen-fixing bacterium Pantoea sp. from sugarcane,” J. Appl Microbiol, vol, 97, pp.504-511, 2004.

[8] R.M. Reis, P.E. Santos, S. Tenoeio-Salgado, J. Vogel, M. Stoffels, S. Guyon, P. Mavingui, V.L. Baldani, M. Schmidt, J.I. Baldani, J. Balandreau, A. Hartmann and J.Caballero-Mellado, "Burkholderia tropica sp. nov., a novel nitrogen-fixing, plant-associated bacterium," Tnt J Syst Evol Microbiol, vol, 54, pp.2155-2162, 2004.

[9] J.Caballero-Mellado, L. Martinez-Aguilar, G. Paredes- Valdez, and P. E. Santos, "Burkholderia unamae sp nov., a $\mathrm{N}_{2}$-fixing rhizospheric and endophytic species. Tnt J Syst Bacteriaol, vol. 54, pp.1165-1172, 2004.

[10] L. Perin, L. Martinez-Aguillar, R. Castro-Gonzalez, P. Estrada-de Los Santos, T. Cabellos-Avelar, H.V. Guedes, V.M. Reis, J. CaballeroMellado,"Diazotrophic Burkholderia species associated with field-grown maize and sugarcane," Appl. Environ. Microbiol., vol. 72, pp:3103-3110, 2006.

[11] M. Rosenblueth and E. Martínez-Romero, "Bacterial endophytes and their interactions with hosts," Molecular Plant-Microbe Interactions., vol. 19, pp.827-837, 2006.

[12] P. Gyaneshwar, E.K. James, N. Mathan, P.M. Reddy, B. Reinhold-Hurek and J.K. Ladha,"Endophytic colonization of rice by a diazotrophic strain of Serratia marcescens," J. Bacteriol., vol.183, pp: 2634-2645, 2001.

[13] N. Weyens, D. van der Lelie, T. Artois, K. Smeets, S. Taghavi, L. Newman, R. Carleer, J. Vangrontsveld,"Bioaugmentation with engineered endophytic bacteria improves contaminant fate in phytoremediation," Environ. Sci. Technol., vol. 43, pp: 9413-9418, 2009.

[14] V.A. Duong "Báo cáo chuyên đề: Thực Trạng sản xuất, yếu tố hạn chế và đề xuất gói kỹ thuật sản xuất mía năng suất chất lượng cao cho vùng Đông Nam Bộ. Hội thảo định hướng nghiên cứu phát triển mía đường bền vững ở Việt Nam, ngày 29/10/2014” (Vietnamese).

[15] L.H.A. Thi, "Report of Sugarcane production in 2012 (Vietnamese)," Department of Agriculture and Rural Development, Vietnam 2013.

[16] N. Tejera, C. Lluch, M.V. Martinez-Toledo and J. Gonzalez-Lopez,"Isolation and characterization of Azotobacter and Azospirillum strains from the sugarcane rhizosphere," Plant Soil., vol. 270, pp:223-232, 2005.

[17] A. Oak, “A reevaluation of nitrogen assimilation in roots,"BioSci., vol. 42, pp.103-111, 1992.

[18] C. Taule, C. Mareque, C. Barlocco, F. Hackembruch, V.M. Reis, M. Sicardi and F. Battistoni,"'The contribution of nitrogen fixation to sugarcane (Saccharum officinarum L.), and the identification and characterization of part of the associated diazotrophic bacterial commnunity," Plant Soil., vol. 356, pp:35-46, 2012.

[19] R. Mendes, A.A. Pizziranni-Kleiner, A.L. Araujo, J.M. Raaijmakers, "Diversity of cultivated endophytic bacteria from sugarcane: genetic and biochemical characterization of Burkholderia cepacia complex isolates," Appl. Environ. Microbiol., vol. 73(22), pp. 7259-7267, 2007.

[20] J.E.Beringer "R factor transfer in Rhizobium legumino-sarum," J. Gen Microbiol. Vol. 84, pp.188-198, 1974.

[21] V.M.Reis, V.M., Olivares, F.L., Oliveira, A.L.M. de, Reis Junior, F.B. dos, Baldani,J.I., Döbereiner, J, "Technical approaches to inoculate micropropa-gated sugar cane plants were Acetobacter diazotrophicus," Plant and Soil, vol. 206, pp.205-211, 1999.

[22] S.A. Gordon and R.P. Weber., "Colometric estimation of indolacetic acid," Plant Physiol., vol 26, pp.192-195, 1951.

[23] B., Schwyn, and J.B. Neilands. "Universal chemical assay for the detection and determination of siderophores". Analytical Biochemistry, vol. 60(1), pp. 47-56, 1987.

[24] B. Neumann, A. Pospiech, and H.U. Schairrer, "Rapid isolation of genomic DNA from Gram-negative," Trends Gent., vol. 8, pp:332333, 1992.

[25] K.D. Zinniel, P. Lambercht, N.P. Harris, Z. Feng, D. Kuczmarshki, P. Higley, C.A. Ishimaru, A. Arunakumari, R.G. Barletta, and A.K Vidaver, "Isolation and charcaterization of endophytic bacteria from agronomic crops and prairie plants", Appl. Environ. Microbiol., vol. 68, pp.2198-2208, 2002.

[26] K. Tamura, D. Peterson, N. Peterson, G Stecher, M. Nei, and S. Kumar, "MEGA5: Molecular Evolutionary Genetics Analysis using Maximu m Likehood, Evolutionary Distance and Maximum Parsimony Methods," Mol. Biol. Evol., vol. 28, pp: 2731-2739, 2011.

[27] M.K. Halushka, J.B. Fan, K. Bentley, L. Hsie, N. Shen, A. Weder, R. Cooper, R. Lipshutz, and A. Charavarti, "Patterns of single-nucleotide polymorphisms in candidate genes for blood-pressure homestasis," Nat. Genet., vol.22(3), pp:239-247, 1999.

[28] O.B. Weber, V.L.D. Baldani, K.R.S. Teixeira, G. Kirchof, J.I. Baldani, and J. Dobereiner, "Isolation and characterization of diazotrophic bacteria from banana and pineapple plants," Plant and Soil., vol 210, pp:103-113, 1999.

[29] N.T.Thu Ha, H.T. Toan, C.N.Diep, "Isolation anf characrterization of endophytic bacteria in several forage grass cultivars," J. Biotechnology., vol. 7(2), pp:241-250, 2009.

[30) H.M. Tam and C.N. Diep, "Isolation, Characterization and Identification of Endophytic Bacteria in Sugarcane (Saccharum spp. L.) Cultivated on Soils of the Dong Nai province, Southeast of Vietnam," American J. Life Science, vol.2, No.2 2014, pp. 361-368. doi: 10.11648/j.ails.2014206.16.

[31] G.A. Watterson, "On the number of segregation sites in general models without recobination," Theor. Pop. Biol., vol. 7, pp. 256-276, 1975.

[32] J. Rozas, and R. Rozas, "DnaSP version 4.1: an integrated program for molecular population genetics and molecular evolution analysis," Bioinformatics, vol. 15, pp: 174-175, 2005.

[33] S.C. Bellone, and C.H. Bellone, "Presence of endophytic diazotrophs in sugarcane juice," World J. Microbiol. Biotechnol., vol.22, pp.1065-1068, 2006.

[34] C.H. Bellone, S.C. Bellone, R. Pedraza, and M.A. Monzon, "Cell colonization and infection thread formation in sugar cane roots by Acetobacter diazotrophicus," Soil Biol. Biochem., vol. 29. pp. 965-967, 1997. 


\section{International Journal of Innovations in Engineering and Technology (IJIET) \\ http://dx.doi.org/10.21172/ijiet.83.029}

[35] G..S.Magnani, C.M. Didonet, L.M.Cruz, C.F.Picheth, F.O.Pedrosa, and E.M.,’Diversity of endophytic bacteria in Brazilian sugarcane,” Genetics and Molecular Research, vol.9(1), pp:250-258, 2010.

[36] A.L.M. Oliveira, S. Urquiaga, J. Döbereiner, J.I. Baldani, The effect of inoculating endophytic N2-fixing bacteria on micropropagated sugarcane plants, Plant Soil, vol.242, pp:205-215, 2002.

[37] R. Dixon, and D. Kahn, "Genetic regulation of biological nitrogen fixation,” Nat. Rev. Microbiol., vol.2, pp: 621-631, 2004.

[38] J.I. Baldani, V.L.D. Baldani, L. Seldin and J. Dobereiner,"Characterization of Herbaspirillum seropedicae gen. nov. sp. nov., a rootassociated nitrogen-fixing bacterium,” Int. J. Syst. Bacteriol., vol. 36, pp: 86-93, 1986.

[39] F.B. Reis Junior, L.G. Silva, V.M. Reis and J. Dobereiner,"Occurrence of diazotrophic bacteria in different sugarcane genetypes," Pesp. Agropec. Bras., vol. 35, pp:985-994, 2000.

[40] A.Suman, A.K. Shasany, M. Singh and H.N. Shani,"Molecular assessment of diversity among endophytic diazotrophs isolated from subtropical Indian sugarcane,” World J. Microbiol. Biotechnol,, vol.17, pp:39-45, 2001.

[41] G.S.Magnani, C.M. Didonet, L.M.Cruz, C.F.Picheth, F.O.Pedrosa, and E.M.,'Diversity of endophytic bacteria in Brazilian sugarcane," Genetics and Molecular Research, vol.9(1),pp:250-258, 2010.

[42] K.U.Zakria, T. Ogawa, A. Yamamoto, Y. Saeki and S. Akao,"Influence of inoculation technique on the endophytic colonization of rice by Pantoea sp. isolated from sweet potato and by Enterobacter sp. isolated from sugarcane Muhammad," Soil Sci. Plant. Nutr., vol. 54, pp:224-236, 2008.

[43] R.J. Rennie, J.R. De Freitas, A.P. Ruschel and P.B. Vose, "Isolation and identification of N2-fixing bacteria associated with sugarcane (Saccharum sp.," Can. J. Microbiol., vol. 28, pp:462-467, 1982.

[44] A.B. Torres, et al.,"Diversity of endophytic enterobacteria associated with different host plants," J. Microbiol., vol. 46, pp:373-379, 2008.

[45] M. Rossignol, A. Basset, O. Espeli, and F. Bocard, "NKBOR, a miniTn-10-based transposon for random insertion in the chromosome of Gram-negative bacteria and the rapid recovery of sequences flanking the insertion sites in Escherichia coli," Res. Microbiol., vol.152, pp:481-485, 2001

[46] C.A. Asis and C.A. Adachi,"'Isolation of endophytic diazotroph Pantoea agglomerans and nondiazotroph Enterobacter asburiae from sweetpotato stem in Japan," Lett. Appl. Microbiol., vol. 38, pp:19-23, 2004.

[47] S. Ruppel, C. Hecht-Buchholz, R. Remus, U. Artmann and R. Schmeizer, "Settlement of the diazotrophic, phytoeffective bacterial strain Pantoea agglomerans on and within winter wheat:an investagation using ELISA and transmission electron microscoly," Plant Soil., vol. 145, pp:261-273, 1992.

[48] W.L. Ararujo, W. Maccheroni, C. Aguilar-Vidoso, P.A.V. Baroso, H.O. Saridakis, and J.L. Azevedo,"Variability and interaction between endophytic bacteria and fungi isolated from leaf tissues of citrus rootstocks," Can. J. Microbiol., vol.47, pp:229-236, 2001.

[49] M. Ongena, et al.,"Systematic induction of phytoalexins in cucumber in response to treatments with fluorescent pseudomonads," Plant Pathol., vol.49, pp:523-530, 2000.

[50] T.F. Hsich, H.C. Huang, and R.S.Erickson, "Biological con trol of bacterial wilt of bean using a bacterial endophyte, Pantoea agglomerans," J. Phytopathol., vol. 153, pp:608-614, 2005.

[51] S.C. Verma, A. Singh, S.P. Chowdhury and A.K. Tripathi, "Endophytic colonization ability of two deep-water rice endophytes, Pantoea sp. and Ochrobactrum sp. using green fluorescent protein reporter," Biotechnol. Lett., vol. 26, pp:425-429, 2004.

[52] M.A. Malboobi et al.,"Solubilization of organic and inorganic phosphates by three highly efficient soil bacterial isolates," World J. Microbiol., vol. 25, pp:1471-1477, 2009.

[53] W. Zimmer, B. Hundeshagen, and E. Niederau,"Demonstration of the indopyruvate decarboxylase gene homologous in different auxin producing species of the Enterobacteriaceae," Can. J. Microbiol., vol. 40, pp:1072-1076, 1994.

[54] M.C. Quecine, W.L. Araujo, P.B. Rosetto, A. Ferreira, S. Tsui, P.T. Lacava, M. Moudin, J.L. Azevedo, and A.A.PizziraniKleiner,"Sugarcane Growth Promotion by the Endophytic Bacterium Pantoea agglomerans 33.1," Appl. Environ. Microbiol., vol. 78(21), pp:7511-7518, 2012.

[55] P. Jha and A. Kumar,"Characterization of Novel Plant Growth Promoting Endophytic Bacterium Achromobacter xylosoxidans from Wheat Plant," Microb. Ecol., vol. 58, pp:179-188, 2009. 